\title{
Atendimento de adolescentes na atenção básica de saúde durante a pandemia de Covid 19
}

\section{Care of adolescents in basic health care during the Covid 19 pandemic}

\author{
Emily Tavares de Melo ${ }^{1}$ (D) \\ Ikaro Victor Ferreira Souza ${ }^{2}$ (1) \\ Carolaine Freitas Amorim ${ }^{3}$ (b)
}

João Victor Lima de Oliveira
Miriã Pontes de Albuquerque $^{5}$
Wellington Pompeu Faustino $^{6}$
Ruth Silva Lima da Costa

1-6Centro Universitário Uninorte (Rio Branco). Acre, Brasil.mogahagi2016@outlook.com, ikarovictor69@gmail.com, freitascarolaine28@gmail.com, joricau@hotmail.com, miriapontes19@gmail.com, welingtonpompeu23@hotmail.com ${ }^{7}$ Autora para correspondência. Centro Universitário Uninorte (Rio Branco). Acre, Brasil. ruttylyma@gmail.com

\begin{abstract}
RESUMO | OBJETIVO: Descrever o atendimento à saúde de adolescentes em uma unidade de saúde do Acre durante a pandemia de COVID 19. MÉTODO: Estudo transversal, exploratório, realizado junto a adolescentes em busca de atendimento em uma unidade de saúde. RESULTADOS: A maioria encontrava-se com 16 anos (40\%), solteiros (90\%), cor da pele parda (55\%), renda familiar de 1 salário mínimo (63\%), ensino médio incompleto (55\%). Não exerciam trabalho remunerado (88\%), não frequentavam religião (60\%), moravam com os pais (75\%), quando buscaram atendimento obtiveram êxito (100\%). Dentre os motivos da busca por atendimento, destacou-se a consulta médica (35\%), seguido da realização de exames (33\%). Alegaram não possuir nenhum problema de saúde pré-existente (98\%) e que não haviam participado de ação educativa nos últimos 12 meses (100\%). Afirmaram não conhecer o agente comunitário de saúde de sua área de abrangência e que ele não realizava as visitas domiciliares regularmente (100\%). A maior parte recebeu orientações dos profissionais de saúde durante o atendimento (80\%) e achavam que as ações da unidade voltadas para os adolescentes eram satisfatórias $(48,0)$. CONCLUSÃO: De forma geral, os adolescentes conseguiram acessar os serviços de saúde no seu loco região durante a pandemia e estavam satisfeitos com o atendimento.
\end{abstract}

DESCRITORES: Atenção Primária em Saúde. Adolescentes. Atendimento. COVID-19
ABSTRACT | OBJECTIVE: To describe the health care provided to adolescents at a health unit in Acre during the COVID 19 pandemic. METHOD: Cross-sectional, an exploratory study carried out with adolescents seeking care at a health unit. RESULTS: Most were 16 years old (40\%), single (90\%), brown skin color (55\%), family income of 1 minimum wage (63\%), incomplete high school (55\%). They did not have a paid job $(88 \%)$, did not attend religion (60\%), lived with their parents (75\%); when they sought care, they were successful (100\%). Among the reasons for seeking care, the medical consultation (35\%) was highlighted, followed by exams (33\%). They claimed that they did not have any pre-existing health problems (98\%) and had not participated in an educational activity in the last 12 months (100\%). They stated that they did not know the community health agent in their coverage area and that he did not regularly carry out home visits (100\%). Most received guidance from health professionals during care $(80 \%)$ and thought that the unit's actions aimed at adolescents were satisfactory (48.0). CONCLUSION: Overall, adolescents could access health services in their local region during the pandemic and were satisfied with the care.

DESCRIPTORS: Primary Health Care. Adolescents. Service. COVID-19 


\section{Introdução}

No Brasil, o Estatuto da Criança e do Adolescente (ECA) considera criança a pessoa até 12 anos de idade incompletos e define a adolescência como a faixa etária de 12 a 18 anos de idade e, em casos excepcionais, e quando disposto na lei, o estatuto é aplicável até os 21 anos de idade. ${ }^{1}$

A adolescência é considerada uma etapa evolutiva peculiar na vida do ser humano, pois configura-se como um momento crucial do seu desenvolvimento, através das mudanças que ocorrem na imagem corporal, mas também a estruturação final da personalidade, não se podendo, portanto, dissociar a adolescência dos seus aspectos biológicos, psicológicos, sociais e culturais.?

Mediante a isso e à necessidade de acompanhamento integral da saúde do adolescente pelas equipes de atenção primária em saúde (APS), dentro do contexto da integralidade da atenção, se faz necessário que as ações de saúde voltadas a eles não sejam apenas transitórias e pontuais, mas que englobem a realidade que os cerca, bem como suas necessidades individuais, principalmente as direcionadas para prevenção e controle dos agravos. ? $^{3}$

No entanto, algumas ações desenvolvidas pelas equipes de saúde e voltadas a esse público alvo podem estar limitadas ao acolhimento e escuta dos mesmos, se fazendo necessário a implementação de ações mais concretas que possam ir de encontro com as necessidades específicas desse grupo, além da necessidade de qualificação das equipes para um olhar diferenciado aos adolescentes. ${ }^{4}$
Nesse contexto, um estudo realizado em uma unidade de saúde da família do município de Olinda-PE, com adolescentes em busca de atendimento, evidenciou que a procura foi predominantemente de natureza curativa e que o número de consultas realizadas não estava adequado ao recomendado pelo Ministério da Saúde, uma vez que esse estabelece que o adolescente realize no mínimo de uma consulta médica e duas consultas de enfermagem por ano. 2.5

Destarte, enfatiza-se que, apesar dos avanços e aumento da cobertura das equipes de atenção primária em saúde no país, ainda persistem grandes desafios no que se refere à qualidade da atenção à população adolescente, que ainda encontra muitas barreiras de acesso aos serviços de saúde. ${ }^{6}$

Frente $a$ isso, a atual pandemia de COVID 19 vem ocasionando uma crise sanitária, política, econômica e social em todo o mundo, exigindo que as ações de saúde culminassem para a necessidade de uma abordagem mais territorializada e domiciliar, sendo essas as características e potencialidades da atenção primária em saúde que desempenham um importante papel na rede assistencial de cuidados, potencialidade essa, que se faz necessária no enfrentamento de qualquer problema de saúde, principalmente frente às epidemias. $\underline{7}$

Dessa forma, torna-se importante evidenciar o contexto do atendimento ao adolescente nas unidades de saúde durante a pandemia de COVID-19, para que se possam tomar medidas necessárias para garantir $\mathrm{o}$ atendimento integral e resolutivo. Mediante a isso, descrever o atendimento à saúde de adolescentes em uma unidade de saúde do Acre, durante a pandemia de COVID 19. 


\section{Métodos}

Trata-se de um estudo transversal descritivo, exploratório, desenvolvido junto a adolescentes de uma unidade de saúde do Acre, durante a pandemia de COVID 19. A amostra foi composta por 40 sujeitos, sendo todos eles adolescentes.

Foram incluídos adolescentes entre 10 a 19 anos, cadastrados na unidade de saúde, que residiam há mais de 6 meses na área de abrangência da unidade, e que buscaram atendimento de saúde na unidade durante a pandemia. Foram excluídos os adolescentes que residiam na área de abrangência, mas que não estivessem cadastrados na unidade de saúde ou residissem no local há menos de seis meses.

O método de seleção da amostra se deu pela amostragem aleatória simples, em que os participantes foram identificados previamente através de registros de cadastros e atendimentos na unidade de saúde, e após selecionados, os pesquisadores entraram em contato prévio com os mesmos por via telefônica, para marcar uma visita à sua residência no melhor dia e horário para a coleta de dados.

A coleta ocorreu nos meses de março e abril de 2021, respeitando o protocolo de medidas impostas pelas autoridades sanitárias locais, para o controle da pandemia, como o distanciamento, uso de máscaras e álcool em gel.

Foi aplicado um instrumento de coleta de dados, elaborado pelos próprios pesquisadores, contendo questões sobre os dados sociodemográficos dos participantes, como sexo, faixa etária, estado civil, escolaridade, renda familiar, religião, além dos motivos para a busca do atendimento e a dinâmica destes, no âmbito da atenção primária, bem como a avaliação dos serviços voltados para a atenção ao adolescente oferecidos na unidade de saúde.

A coleta só teve início após a leitura explicativa e assinatura do Termo de assentimento Livre e Esclarecido (TALE) e do Termo de Consentimento Livre e Esclarecido (TCLE).

Após essa etapa, os dados foram analisados criteriosamente quanto às interligações de todas as variáveis e foram apresentados em frequência absoluta e percentual e demostrados em forma de tabelas e gráficos de acordo com as variáveis existentes. Para produção dos gráficos foi utilizada a ferramenta do Microsoft Office Excel 2010.

A pesquisa foi aprovada pelo comitê de ética em pesquisa local, através do parecer $n^{\circ} 4.586 .534$.

\section{Resultados}

Os dados evidenciados na Tabela 1 demostram que em relação à idade, observou-se que a maioria dos adolescentes se encontrava com 16 anos (40\%). Quanto ao estado civil, eram solteiros $36(90 \%)$, cor da pele parda $22(55 \%)$, e a renda familiar era de 1 salário mínimo 25(63\%). Quanto à escolaridade, a maior parte possuía o ensino médio incompleto 22 (55\%), não exerciam nenhum trabalho remunerado 35 (88\%), não frequentavam nenhuma religião 24 (60\%) e ainda moravam com os pais 30 (75\%). 
Tabela 1. Perfil sócio demográfico de adolescentes que buscaram atendimento em uma unidade básica de saúde no Acre em 2021 ( $n=40$ )

\begin{tabular}{|c|c|c|}
\hline Variável & $\mathbf{N}$ & $\%$ \\
\hline \multicolumn{3}{|l|}{ Faixa Etária } \\
\hline 15 & 1 & $3 \%$ \\
\hline 16 & 2 & $5 \%$ \\
\hline 17 & 16 & $40 \%$ \\
\hline 18 & 12 & $30 \%$ \\
\hline 19 & 9 & $23 \%$ \\
\hline \multicolumn{3}{|l|}{ Estado Civil } \\
\hline Solteiro & 36 & $90 \%$ \\
\hline União Estável & 4 & $10 \%$ \\
\hline \multicolumn{3}{|l|}{ Cor da Pele } \\
\hline Parda & 22 & $55 \%$ \\
\hline Branca & 9 & $23 \%$ \\
\hline Negra & 9 & $23 \%$ \\
\hline \multicolumn{3}{|l|}{ Sexo } \\
\hline Feminino & 38 & $95 \%$ \\
\hline Masculino & 2 & 0,05 \\
\hline \multicolumn{3}{|l|}{ Renda Familiar } \\
\hline Menos de 1 Salário & 14 & $35 \%$ \\
\hline 1 Salário Mínimo & 25 & $63 \%$ \\
\hline Mais de 1 Salário Mínimo & 1 & $3 \%$ \\
\hline \multicolumn{3}{|l|}{ Escolaridade } \\
\hline Ensino Fundamental Incompleto & 1 & $3 \%$ \\
\hline Ensino Médio Incompleto & 22 & $55 \%$ \\
\hline Ensino Médio Completo & 17 & $43 \%$ \\
\hline \multicolumn{3}{|l|}{ Trabalho remunerado } \\
\hline Sim & 5 & $13 \%$ \\
\hline Não & 35 & $88 \%$ \\
\hline \multicolumn{3}{|l|}{ Religião } \\
\hline Sem Religião & 24 & $60 \%$ \\
\hline Católica & 4 & $10 \%$ \\
\hline Evangélica & 12 & $30 \%$ \\
\hline \multicolumn{3}{|l|}{ Com quem reside } \\
\hline Pais & 30 & $75 \%$ \\
\hline Mãe & 5 & $13 \%$ \\
\hline Pai & 1 & $3 \%$ \\
\hline Com Companheiro & 4 & $10 \%$ \\
\hline
\end{tabular}

Observou-se que todos os adolescentes 40 (100\%) que buscaram atendimento na unidade básica de saúde obtiveram êxito. Dentre os motivos da procura por atendimento, destacou-se a consulta médica 14 (35\%), seguido de realização de exames 13 (33\%). No que diz respeito aos problemas de saúde, a maioria 39 (98\%) alegou não apresentar nenhuma doença pré-existente, sendo que nenhum deles 40 (100\%) alegou ter participado de ação educativa nos últimos 12 meses, pois as mesmas estavam suspensas por conta da pandemia 39 (98\%). No que se refere ao contato com o agente comunitário de saúde da área de abrangência de sua residência, eles afirmaram que não o conheciam e alegaram que ele não visitava sua casa regularmente, 40 (100\%) (Tabela 2). 


\begin{tabular}{|c|c|c|}
\hline Variável & $\mathbf{N}$ & $\%$ \\
\hline \multicolumn{3}{|c|}{ Procurou atendimento nos últimos 12 meses? } \\
\hline $\operatorname{Sim}$ & 40 & $100 \%$ \\
\hline Não & 0 & 0 \\
\hline \multicolumn{3}{|l|}{ Conseguiu ser atendido? } \\
\hline Sim & 40 & $100 \%$ \\
\hline Não & 0 & 0 \\
\hline \multicolumn{3}{|l|}{ Motivo da consulta? } \\
\hline Consulta Médica & 14 & $35 \%$ \\
\hline Pré-Natal & 10 & $25 \%$ \\
\hline Realização de exames & 13 & $33 \%$ \\
\hline Vacina & 3 & $8 \%$ \\
\hline \multicolumn{3}{|l|}{ Possui algum problema de saúde? } \\
\hline Sim (Asma) & 1 & $3 \%$ \\
\hline Não & 39 & $98 \%$ \\
\hline \multicolumn{3}{|c|}{ Participou de atividade educativas nos últimos 12 meses? } \\
\hline Sim & 0 & 0 \\
\hline Não & 40 & $100 \%$ \\
\hline \multicolumn{3}{|l|}{ Se não porquê? } \\
\hline Estão suspensas por conta da pandemia & 39 & $98 \%$ \\
\hline Não sei porque sou novo no local & 1 & $3 \%$ \\
\hline \multicolumn{3}{|c|}{ Você conhece o agente comunitário de saúde da sua área? } \\
\hline Sim & 0 & 0 \\
\hline Não & 40 & $100 \%$ \\
\hline \multicolumn{3}{|l|}{ Ele visita sua casa regularmente? } \\
\hline Sim & 0 & 0 \\
\hline Não & 40 & $100 \%$ \\
\hline
\end{tabular}

Os dados apresentados na tabela 3 demostram que a maioria recebeu orientações dos profissionais de saúde durante o atendimento recebido na unidade de saúde (80\%). Quando questionados sobre o fato de a unidade oferecer ações voltadas para a educação em saúde do adolescente 25 (63\%), responderam que sim, mas não sabiam informar quais, pois no momento estavam suspensas por conta da pandemia 40 (100\%). A maior parte evidenciou que os atendimentos voltados para os adolescentes ofertados pela equipe da unidade eram satisfatórios 19 (48\%) e que não precisava melhorar 37 (93\%).

Tabela 3. Avaliação dos atendimentos prestados aos adolescentes em uma unidade básica de saúde no Acre, em 2021, na visão dos adolescentes (n=40)

\begin{tabular}{|c|c|c|}
\hline Variável & $\mathbf{N}$ & $\%$ \\
\hline \multicolumn{3}{|c|}{ Durante o atendimento na unidade recebeu alguma orientação dos profissionais de saúde? } \\
\hline $\operatorname{Sim}$ & 32 & $80 \%$ \\
\hline Não & 8 & $20 \%$ \\
\hline \multicolumn{3}{|l|}{ A unidade de saúde oferece ações voltadas para educação em saúde? } \\
\hline Sim & 25 & $63 \%$ \\
\hline Não & 15 & $38 \%$ \\
\hline \multicolumn{3}{|l|}{ Quais as atividades oferecidas? } \\
\hline Não sei pois no momentos estão suspensas devido a pandemia & 40 & $100 \%$ \\
\hline \multicolumn{3}{|c|}{ O que você acha do atendimento oferecido na unidade para os adolescentes? } \\
\hline Ótimo & 9 & $23 \%$ \\
\hline Bom & 19 & $48 \%$ \\
\hline Regular & 11 & $28 \%$ \\
\hline Poderia Melhorar & 1 & $3 \%$ \\
\hline \multicolumn{3}{|l|}{ Como $o$ atendimento pode melhorar? } \\
\hline Não acho que precisa melhorar & 37 & $93 \%$ \\
\hline Respeitar as prioridades & 2 & $5 \%$ \\
\hline Passar mais informações & 1 & $3 \%$ \\
\hline
\end{tabular}




\section{Discussão}

Estudos sobre a busca por atendimentos em serviços de atenção primária à saúde entre adolescentes são pouco frequentes no Brasil, sendo a maioria voltados para um determinado tipo de cuidado, como gravidez na adolescência, saúde bucal e saúde mental-9 o que tornou limitada as discussões dos aspectos demográficos dos adolescentes participantes do presente estudo.

Nesse sentido, essa carência reforça a necessidade da realização de mais estudos nesse âmbito, a fim de contribuir com achados que subsidiem o comportamento dos adolescentes e suas relações com as práticas assistenciais dos serviços, principalmente relacionados ao seu perfil sociodemográfico. ${ }^{10}$

Mediante a isso, um estudo realizado com 812 indivíduos de 15 a 24 anos de idade, sobre o acesso aos serviços de atenção primária à saúde por adolescentes e jovens em um município do estado da Bahia, evidenciou que, quanto às características demográficas, uma maior proporção de indivíduos pertenciam ao sexo feminino $(67,4 \%)$, de $15-19$ anos $(55,7 \%)$ e cor da pele autor referida parda (90,9\%); além disso, $67,1 \%$ não trabalhavam, corroborando com os achados desta pesquisa. 11

Chama-se atenção para o fato de que a maioria dos adolescentes em busca de atendimento pertenciam ao sexo feminino, o que pode ser justificado pelo fato de que elas, ao alcançarem a puberdade e com a iniciação sexual, procuram com maior frequência os serviços de saúde, principalmente os atendimentos ginecológicos. ${ }^{12}$

A priori, na APS, são realizados os cuidados básicos quanto aos problemas mais comuns, voltados para a promoção e prevenção à saúde, sendo neste momento que ocorrem à abordagem individual e familiar, a fim de estabelecer estratégias de promoção e prevenção à saúde. Dentro da APS, estão as unidades básicas de saúde (UBS), caracterizadas por se localizarem próximas às casas dos indivíduos, com o intuito de garantir um maior acesso da população aos serviços de saúde oferecidos. ${ }^{13}$
Dessa forma, a procura por serviços de saúde pode ser influenciada tanto pelas características e necessidades dos indivíduos quanto pela oferta dos atendimentos, além da facilidade de acesso, disponibilidade e vinculação com os profissionais de saúde. ${ }^{14}$

Sendo assim, em uma pesquisa realizada sobre a saúde do adolescente escolar, verificou-se que $48 \%$ dos participantes procurou algum serviço ou profissional de saúde nos últimos 12 meses anteriores à pesquisa, sendo a atenção básica o serviço mais referido $(47,5 \%)$. Entre aqueles que procuraram a unidade, $85,1 \%$ afirmaram ter obtido atendimento com êxito ${ }^{15}$, resultados esses que corroboram com os achados do presente estudo.

Referente aos motivos pela busca por atendimento durante a pandemia, os achados evidenciaram a consulta médica como principal motivo. Dados da literatura demostram que as queixas mais comuns que levam os adolescentes à consulta médica, no dia a dia das unidades, são os relacionados ao crescimento e desenvolvimento, os problemas relacionados à puberdade, excesso de peso, transtornos alimentares, dores recorrentes, distúrbios menstruais, acnes, desvios de coluna, dificuldades escolares e relacionamentos familiares, e questões de saúde mental cada vez mais frequentes. ${ }^{16}$

No que se refere à oferta de ações educativas oferecidas pela unidade, chama-se atenção para o fato das atividades coletivas estarem suspensas devido à pandemia, o que levou a equipe a repensar estratégias de educação em saúde individuais durante os atendimentos. O que é de extrema importância, pois a educação em saúde é uma estratégia para a formação da identidade do adolescente, levando-o a refletir sobre suas práticas e a desenvolverem novos comportamentos. ${ }^{17}$ Também é um momento oportuno para orientá-los sobre as medidas de prevenção e controle impostas e necessárias para o enfrentamento da pandemia.

Sendo assim, essas ações, mesmo que pontuais e realizadas de forma individual, podem apresentar resultados positivos quanto ao aumento de conhecimento dos adolescentes nas temáticas de interesse para eles e para que se tornem sujeitos mais críticos e conscientes dos seus atos. $\frac{18}{18}$ 
Frente à atuação do agente comunitário de saúde (ACS), o Ministério da Saúde adverte que esse é o profissional que dever estar mais próximo a população, pois é o trabalhador da saúde que possui maior conhecimento das situações de vida das famílias, incluindo os adolescentes, identificando-os e acompanhando o seu crescimento e desenvolvimento, considerando que é necessário orientá-los quanto à necessidade de vacinação, à sexualidade, o uso de álcool e outras drogas, à importância da educação, à violência e acidentes, aos riscos no trânsito, à atividade física e saúde, aos hábitos saudáveis, à saúde bucal. $19-20$

No entanto, observa-se que, no presente estudo, essas ações não estão sendo efetivas, uma vez que, por unanimidade, os adolescentes afirmaram não conhecer o ACS de sua área e nem receber visita regularmente para o devido acompanhamento; fato esse que deve ser revisto pela coordenação da unidade de saúde onde esse adolescente é cadastrado e que, apesar da necessidade de distanciamento social impostas pela pandemia, novas estratégias de acompanhamento devem ser idealizadas, como por exemplo o contato através de aplicativos de mensagens, para garantir que não haja interrupção das ações previstas.

Referente a isso, salienta-se que a adolescência é uma fase da vida, marcada por intensas transformações tanto físicas quanto psicológicas, o que pode levar, dependendo do contexto e das situações vivenciadas, a torná-los vulneráveis a inúmeros riscos, se fazendo então necessária a orientação contínua por parte dos profissionais de saúde e familiares, na tentativa de torná-los menos vulneráveis. ${ }^{21}$

Um outro aspecto a ser levado em conta é que as atividades de saúde, mesmo suspensas por conta da pandemia, devem continuar sendo realizadas de forma individual durante a visita dos adolescentes à unidade de saúde, a fim de garantir a continuidade das ações desenvolvidas em educação em saúde, tão primordial para o crescimento e desenvolvimento saudável desses indivíduos. ${ }^{22}$

Chama-se atenção para o fato de que a maioria demonstrou satisfação com o atendimento voltado para eles na unidade de saúde, o que é benéfico, uma vez que na atual organização do sistema de saúde, a atenção primária constitui-se como a porta de entrada no sistema e, nesse sentido, permite o acesso do adolescente; e o fato dele se sentir satisfeito como atendimento, certamente o levará a retornar à unidade para novos atendimentos. $\underline{\underline{23}}$

Assim, um estudo realizado sobre as perspectivas dos adolescentes sobre os profissionais e os serviços de saúde, as preferências, barreiras e satisfação, evidenciou que a maioria demostrou satisfação com o atendimento ofertado pelos profissionais por estes terem se mostrado simpáticos, acolhedores e competentes. .4

Sendo assim, pelo fato de a adolescência se tratar de uma fase com predomínio de higidez física, é comum a ausência de demanda espontânea em instituições de atenção básica à saúde e, consequentemente, um despreparo do serviço de saúde em atendê-los. ${ }^{25}$

Por isso, torna-se importante a qualificação do profissional para atender esse público alvo, aproximando-se das suas necessidades, promovendo o acolhimento e favorecendo o vínculo, afim de garantir o atendimento de suas necessidades.

Dentre as limitações do estudo, destaca-se o tamanho amostral, já que uma mostra pequena pode dificultar o encontro de relações e generalizações significativas a partir dos dados. Outra limitação foi o fato de a pesquisa ter sido coletado no período de pandemia de COVID-19 no Brasil, o que pode ter levado a uma menor procura por atendimentos de saúde devido as medidas de isolamento social impostas.

\section{Conclusão}

Esta pesquisa evidenciou que os adolescentes conseguiram acessar os serviços de atenção primária à saúde no seu loco região durante a pandemia de COVID 19, que não apresentavam sérios problemas de saúde e que estavam satisfeitos com o atendimento prestado pelos profissionais de saúde na unidade, pois, apesar das atividades educativas estarem suspensas devido a pandemia, os profissionais estavam realizando orientações individuais durante os atendimentos. 
No entanto, chama-se atenção para o fato de que essa pode não ser a realidade de todos eles. A parte dos estudos pesquisados evidenciou que existem dificuldades de acesso da maioria deles às unidades de saúde. Desta maneira, faz-se necessário fortalecer políticas públicas já existentes e repensar as práticas das equipes de saúde de forma a preconizar uma atenção acolhedora e de qualidade para esse público alvo.

\section{Contribuições dos autores}

Melo ET e Souza IVF participaram da concepção, delineamento, coleta de dados da pesquisa, interpretação, busca e análise dados e redação do artigo científico. Amorim CF, Oliveira JVL, Albuquerque MP e Faustino WP participaram da coleta e interpretação de dados da pesquisa. Costa RSL participou da concepção, delineamento, submissão do projeto ao Comitê de ética e pesquisa, interpretação dos dados, busca e análise estatística dos dados da pesquisa, interpretação dos resultados e redação do artigo científico.

\section{Conflito de interesses}

Nenhum conflito financeiro, legal ou político envolvendo terceiros (governo, empresas e fundações privadas, etc.) foi declarado para nenhum aspecto do trabalho submetido (incluindo, mas não se limitando a subvenções e financiamentos, participação em conselho consultivo, desenho de estudo, preparação de manuscrito, análise estatística, etc.).

\section{Referências}

1. Eisenstein E. Adolescência: definições, conceitos e critérios. Adolesc Saúde [Internet]. 2005;2(2):6-7. Disponível em: https:// pesquisa.bvsalud.org/portal/resource/pt/abr-451

2. Ministério da Saúde (Brasil), Secretaria de Atenção à Saúde, Departamento de Ações Programáticas e Estratégicas. Proteger e cuidar da saúde de adolescentes na atenção básica [Internet]. 2a ed. Brasília: Ministério da Saúde; 2018. Disponível em: http:// repositorio.asces.edu.br/handle/123456789/1944.

3. Carmo ME, Guizardi FL. O conceito de vulnerabilidade e seus sentidos para as políticas públicas de saúde e assistência social. Cad. Saúde Pública. 2018;34(3):e00101417. https://doi. org/10.1590/0102-311X00101417

4. Leal CBM, Porto AO, Barbosa CB, Fernandes TSS, Pereira ES, Viana TBP. Assistência de Enfermagem ao Público Adolescente na Atenção Primária. Revista Enfermagem Atual. 2019;86(24). https:// doi.org/10.31011/reaid-2018-v.86-n.24-art.123
5. Branco VMC. Desafios do cuidado integral ao adolescente na atenção primária à saúde. Reupe [Internet]. 2021;6(suppl 1):58-9. Disponível em: https://www.revistaextensao.upe.br/index.php/ reupe/article/view/120

6. Silva RF, Engstrom EM. Atenção integral à saúde do adolescente pela Atenção Primária à Saúde no território brasileiro: uma revisão integrativa. Interface. 2020;24(suppl 1). https://doi. org/10.1590/Interface.190548

7. Medina MG, Giovanella L, Bousquat A, Mendonça MHM, Aquino R, Comitê Gestor da Rede de Pesquisa em Atenção Primária à Saúde da Abrasco. Atenção primária à saúde em tempos de COVID-19: o que fazer? Cad Saúde Pública. 2020;36(8). https://doi. org/10.1590/0102-311X00149720

8. Rocha RAC, Goes PSA. Comparação do acesso aos serviços de saúde bucal em áreas cobertas e não cobertas pela Estratégia Saúde da Família em Campina Grande, Paraíba, Brasil. Cad Saúde Pública. 2008;24(12):2871-80. https://doi.org/10.1590/S0102311X2008001200016

9. Quinderé PHD, Jorge MSB, Nogueira MSL, Costa LFA, Vasconcelos MGF. Acessibilidade e resolubilidade da assistência em saúde mental: a experiência do apoio matricial. Ciênc saúde coletiva. 2013;18(7):2157-66. https://doi.org/10.1590/S1413$\underline{81232013000700031}$

10. Silva ZP, Ribeiro MCSA, Barata RB, Almeida MF. Perfil sociodemográfico e padrão de utilização dos serviços de saúde do Sistema Único de Saúde (SUS), 2003- 2008. Ciênc saúde coletiva. 2011;16(9):3807-16. https://doi.org/10.1590/S1413$\underline{81232011001000016}$

11. Martins MMF, Aquino R, Pamponet ML, Pinto Junior EP, Amorim LDAF. Acesso aos serviços de atenção primária à saúde por adolescentes e jovens em um município do Estado da Bahia, Brasil. Cad Saúde Pública. 2019;35(1):e00044718. https://doi. org/10.1590/0102-311X00044718

12. Olsen JM, Lago TG, Kalckmann S, Alves MCGP, Escuder MML. Práticas contraceptivas de mulheres jovens: inquérito domiciliar no Município de São Paulo, Brasil. Cad Saúde Pública. 2018;34(2):e00019617. https://doi.org/10.1590/0102311X00019617

13. Cabral ERM, Bonfada D, Melo MC, Cesar ID, Oliveira RE, Bastos TF, et al. Contribuições e desafios da Atenção Primária à Saúde frente à pandemia de COVID-19. InterAm J Med Health. 2020;3:e202003012. https://doi.org/10.31005/iajmh.v3i0.87

14. Oliveira MM, Andrade SSCA, Stopa SR, Malta DC. Procura por serviços ou profissionais de saúde entre adolescentes brasileiros, segundo a Pesquisa Nacional de Saúde do Escolar de 2015. Rev Bras Epidemiol. 2018;21(suppl 1): e180003.supl.1. https://doi. org/10.1590/1980-549720180003.supl.1 
15. Oliveira M, Andrade S, Campos M, Malta D. Fatores associados à procura de serviços de saúde entre escolares brasileiros: uma análise da Pesquisa Nacional de Saúde do Escolar (PeNSE), 2012. Cad Saúde Pública. 2015;31(8):1603-14. https://doi. org/10.1590/0102-311X00165214

16. Sociedade Brasileira de Pediatria, Departamento Científico de Adolescência. Consulta do adolescente: abordagem clínica, orientações éticas e legais como instrumentos ao pediatra [Internet]. Manual de Orientação. Departamento Científico de Adolescência; 2019. Disponível em: https://www.sbp.com.br/ fileadmin/user_upload/21512c-MO__ConsultaAdolescente_abordClinica orientEticas.pdf

17. Salci MA, Maceno P, Rozza SG, Silva DMGV, Boehs AE, Heidemann ITSB. Educação em saúde e suas perspectivas teóricas: algumas reflexões. Texto contexto - enferm. 2013;22(1):224-30. https://doi.org/10.1590/S0104$\underline{07072013000100027}$

18. Viero VSF, Farias JM, Ferraz F, Simões PW, Martins JA, Ceretta LB. Educação em saúde com adolescentes: análise da aquisição de conhecimentos sobre temas de saúde. Esc Anna Nery [Internet]. 2015;19(3):484-90. Disponível em: https://www.scielo. br/j/ean/a/wyHpK9Nm4p4wjjp7sHKbkLw/abstract/?lang=pt

19. Ministério da Saúde (Brasil), Secretaria de Atenção à Saúde, Departamento de Atenção Básica. Guia prático do agente comunitário de saúde [Internet]. Brasília: Ministério da Saúde; 2009. [citado em 2021 ago. 20]. Disponível em: http://189.28.128.100/dab/docs/publicacoes/geral/guia acs.pdf
20. Brasil EGM, Amorim DU, Queiroz MV. Atuação do agente comunitário de saúde no cuidado ao adolescente: propostas educativas. Adolesc Saúde [Internet]. 2013;10(3):28-35. Disponível em: https://pesquisa.bvsalud.org/portal/resource/pt/abr-338

21. Albuquerque GA, Belém JM, Nunes JFC, Leite MF, Adami F. Orientações familiares: impactos na saúde do adolescente do sexo masculino. Adolesc Saúde. 2016;13(2):67-76.

22. Oliveira CB, Frechiani JM, Silva FM, Maciel ELN. As ações de educação em saúde para crianças e adolescentes nas unidades básicas da região de Maruípe no município de Vitória. Ciênc saúde coletiva. 2009;14(2):635-44. https://doi.org/10.1590/S1413$\underline{81232009000200032}$

23. Costa RF, Queiroz MVO, Zeitoune RCG. Cuidado aos adolescentes na atenção primária: perspectivas de integralidade. Esc Anna Nery. 2012;16(3):466-72. https://doi.org/10.1590/S1414$\underline{81452012000300006}$

24. Graça MGV. Perspectivas dos adolescentes sobre os profissionais e os serviços de saúde: preferências, barreiras e satisfação [dissertação] [Internet]. Lisboa: Universidade de Lisboa; 2016. Disponível em: https://repositorio.ul.pt/handle/10451/25371

25. Silva NT, Bittar NA, Carvalho KCN. Atenção à saúde dos adolescentes: percepção de agentes comunitários de saúde e médicos das unidades básicas de saúde de Anápolis - Goiás. Rev Educ Saúde. 2018;6(2):56-64. http://dx.doi.org/10.29237/23589868.2018v6i2.p56-64 